2017 - Volume: 18 Number: 3

Page: $632-639$

DOI : $10.18038 /$ aubtda. 310298

Received: 03 May 2017 Revised: 30 June 2017 Accepted: 07 July 2017

\title{
ASSESSMENT ON THE ACCURACY OF PIEZOELECTRIC PROPERTY CALCULATIONS OF SINGLE LAYER TWO DIMENSIONAL HEXAGONAL CRYSTALS
}

\author{
Cem SEVİK* \\ Mechanical Engineering Department, Engineering Faculty, Anadolu University, Eskişehir, Turkey
}

\begin{abstract}
The finite difference and the density functional perturbation theory based piezoelectric property calculation methods are applied to the novel two dimensional hexagonal materials named as group II-VI monolayers and transition metal dichalcogenides for the purposes of comparison. The clamped- and relaxed- ion coefficients have been calculated separately to test the accuracy of both methods on electronic and ionic piezoelectric response contributions. While there is no significant difference between the clamped-ion piezoelectric coefficients calculated with these two methods, a notable difference between the values for relaxedion piezoelectric coefficients are determined. Considering the results of the density functional perturbation theory given in the previous applications, it has been determined that the consistency of the finite difference method in the ionic contribution calculation do not provide reliable results for some 2D materials. We have predicted that the atomic relaxation for different strain values is not adequate to achieve accurate results for ionic contribution of piezoelectric coefficient. However, on the contrary to the explicit difference in the coefficients calculated with two different approaches, our results clearly show that the piezoelectric potentials of the considered materials can be determined accurately and reliably by both methods.
\end{abstract}

Keywords: Piezoelectricity, Two-Dimensional Materials, Polarization

\section{INTRODUCTION}

Piezoelectricity is the electric dipole moment that arise in non-centrosymmetric dielectric crystals in response to applied stress [1]. This response, called as the direct piezoelectric effect, is a reversible process and conversely strain generation in a piezoelectric material upon the application of an electric field is called as indirect piezoelectric effect. Piezoelectric materials have been adopted to numerous technological applications and devices such as pressure sensors [2], transducers [3], high voltage generators [4], and energy harvesters [5, 6]. However, research on innovative materials with high piezoelectricity is still of interest to the scientific community.

Recently, peculiar piezoelectric properties of two-dimensional dielectric materials have been demonstrated by both experimental and theoretical studies [7-11]. The relaxed-ion piezoelectric stress $\left(e_{11}\right)$ and piezoelectric strain $\left(d_{11}\right)$ coefficients of single layer hexagonal $\mathrm{BN}$, and transition metal dichalcogenides such as $\mathrm{MoS}_{2}, \mathrm{MoSe}_{2}, \mathrm{WS}_{2}$, and $\mathrm{WSe}_{2}$ have been determined as comparable or even better than those of conventional bulk piezoelectric materials by density functional perturbation theory calculations (DFPT) [7]. Moreover, $e_{11}$ and $d_{11}$ coefficients of $\mathrm{CrS}_{2}$ have been predicted as significantly larger than those of single layer $\mathrm{MoS}_{2}$ [8]. In addition to these theoretical calculations, $e_{11}$ coefficient of monolayer $\mathrm{MoS}_{2}$ has been experimentally measured as $2.9 \times 10^{-10} \mathrm{Cm}^{-1}$, which is in quite good agreement with calculations [12].

Following these studies, the piezoelectric properties of different group III [10], and group IV monochalcogenides [11] have been studied by finite displacement (FD) method based on polarization calculations via density functional theory. Quite surprisingly, the mechanical-electrical energy conversion ratio, $d_{11}$ coefficients of some of these materials has been calculated to be one or two orders of magnitude larger than that of conventional bulk materials such as $\alpha$-quartz $\left(d_{11}=2.3 \mathrm{pmV}^{-1}\right)$, wurtzite- 
$\operatorname{GaN}\left(d_{33}=3.1 \mathrm{pmV}^{-1}\right)$, and wurtzite-AlN $\left(d_{33}=5.1 \mathrm{pmV}^{-1}\right)$. For instance, the values 17.0, 17.1, 8.5, $16.3,21.7,212.1$, and $250.5 \mathrm{pmV}^{-1}$ have been obtained for monolayer crystals, $\mathrm{CaS}, \mathrm{SrSe}$ [13], $\mathrm{CrSe}_{2}$, $\mathrm{CrTe}_{2}$ [8], $\mathrm{CdO}$ [7], GeSe, and $\mathrm{SnSe}_{3}$ [9], respectively. These results clearly indicate the remarkably high piezoelectric properties of non-centrosymmetric dielectric monolayer materials and demonstrate their potential for adaptation to future technological applications such as nanorobotics, piezotronics, and nanoelectromechanical systems.

In addition to the materials mentioned above, there are numerous two dimensional materials that have potential to show high piezoelectric efficiency. Therefore, investigation of piezoelectric properties of these new possible materials by theoretical methods is significantly crucial in order to give an optimum direction to experimental studies. Indeed, the experimental measurements to be made on this field require careful material selection due to the cost and difficulty of nanoscale measurement techniques. In the literature, two different density functional theory based methods are used to calculate the piezoelectric properties of layered materials as mentioned above. In general, there are no significant differences between the values calculated by these two different methods, but in some cases proportional differences of up to two times can be observed $[7,8]$. Therefore, in order to evaluate the calculated values more accurately the comparison of these two methods for different layered materials will make an important contribution to the literature.

With this intention, we have performed a systematic first-principles study to investigate piezoelectric properties of noncentrosymmetric single layer group II-V monolayers ( $\mathrm{NY}$, where $\mathrm{N}=\mathrm{Be}, \mathrm{Mg}, \mathrm{Ca}, \mathrm{Sr}$, $\mathrm{Ba}$ and $\mathrm{Y}=\mathrm{S}, \mathrm{Se}, \mathrm{Te})$ and transition metal dichalcogenides $\left(\mathrm{MX}_{2}\right.$, where $\mathrm{M}=\mathrm{Mo}, \mathrm{W}$ and $\left.\mathrm{X}=\mathrm{S}, \mathrm{Se}, \mathrm{Te}\right)$ by using both FD [14] and DFPT [15] based methods for the first time in the literature.

\section{COMPUTATIONAL METHOD}

Within the frame of this study, first-principles calculations based on density functional-theory (DFT), as implemented in the Vienna Ab initio Simulation package (VASP) code have been performed [16, 17]. The generalized gradient approximation (GGA) within the Perdew-Burke-Ernzerhof (PBE) formulation have been chosen to treat the exchange-correlation interactions [18]. The single electron wave functions have been expanded in plane waves with kinetic energy cutoff of $600 \mathrm{eV}$. For the structure optimizations, Brillouin zone integrations have been carried out using a regular $26 \times 26 \times 1 k$-point mesh within the Monkhorst-Pack scheme [19]. The convergence criterion for total (free) energy change and ionic forces have been set as $10^{-7} \mathrm{eV}$ and $10^{-3} \mathrm{eV}^{-1}$, respectively. To minimize the periodic interaction along the $z$ direction, the vacuum space between the layers has been taken at least $15 \AA$.

The piezoelectric properties have been predicted by two different methods as stated above. First, finite difference [14] method based on the Berry phase approach has been applied, in which the piezoelectric stress coefficients, $e_{i j}$, can be defined in terms of the induced polarization due to a strain $\left(\varepsilon_{j}\right)$ change (for constant temperature, $\mathrm{T}$ and Electric field, E) as follows,

$$
e_{i j}^{T}=\left(\frac{\partial P_{i}}{\partial \varepsilon_{j}}\right)_{E T}
$$

where $P_{i}$ is the induced polarization along the direction $i$ as a result of an applied strain along the direction $j$. For the considered materials, we only need to calculate the $e_{11}$ component of the piezoelectric stress tensor due to their hexagonally symmetric crystal structures [8]. Therefore, $e_{11}$ coefficients of all the crystals have been obtained by polarization calculations as implemented in the VASP package, with applied uniform strain, ranging from 0.01 to -0.01 in steps of 0.005 , along the armchair side of the rectangular cell shown in Figure 1. Here, the ions have been kept in their strained positions or allowed to relax to their new equilibrium positions, and consequently the clamped- and relaxed-ion $e_{11}$ coefficients are predicted, respectively. 


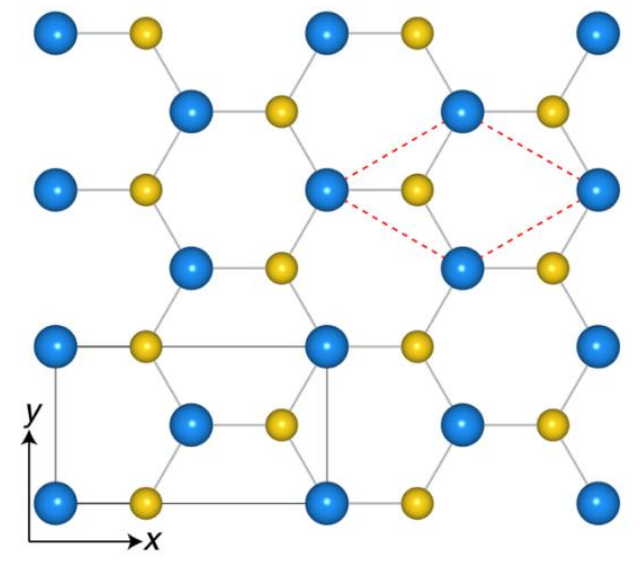

Figure 1. Top views of schematically crystal structures of $\mathrm{MX}_{2}$ (double occupancy at the position shown by yellow) and NY (single occupancy at the position shown by yellow) systems. The red dashed lines show the unit cell of the structures and the black solid lines show the cell used in finite difference calculations.

As a second case, density functional perturbation theory [15] simulations as implemented in the VASP code have been used. In this method, the proper piezoelectric constants, $e_{i j}$, defined by the following thermodynamic derivative (for constant strain and temperature)

$$
e_{i j}^{T}=-\left(\frac{\partial \sigma_{j}}{\partial E_{i}}\right)_{\varepsilon, T}, j \rightarrow\{11,22,33,12,23,31\}, i \rightarrow\{1,2,3\}
$$

where, $\sigma$ denotes the stress tensor $E$ denotes the electric field. The clamped-ion (purely electronic contribution) and relaxed-ion (the sum of ionic and electronic contribution) piezoelectric stress $\left(e_{11}\right)$ tensors have been directly obtained by these calculations [20]. Here, a highly dense $k$-point mesh, $36 \times 36 \times 1$, has been used to accurately predict these tensor components.

The corresponding piezoelectric strain tensor $\left(d_{11}\right)$ of each material has been predicted from the following relation as previously shown by Duerloo et al. [14],

$$
d_{11}=\frac{e_{11}}{\left(C_{11}-C_{12}\right)}
$$

where, $e_{11}$ is the piezoelectric stress coefficients calculated by DFPT or FD and $\mathrm{C}_{\mathrm{xx}}$ are the elastic stiffness coefficients. The used $\mathrm{C}_{\mathrm{xx}}$ coefficients for all the materials considered in this study have been adopted from our previous calculations $[8,13]$. It is important to note that all the reported piezoelectric constants in this study are associated with the pure, defect-free, and strain-free two-dimensional hexagonal crystals, at a temperature of $0 \mathrm{~K}$.

\section{RESULTS and DISCUSSION}

With the intention to test the accuracy of the available approaches mentioned above, we have performed systematic simulations for group II-VI monolayers and transition metal dichalcogenides (TMDCs). Here, all the materials have been determined regarding their band-gap values due to the fact that a piezoelectric material has to be a wide band-gap semiconductor or an insulator to avoid current leakage. Strictly, the intrinsic semiconductor nature of all the materials has been proven by several previous calculations, which show indirect or direct band-gap values as large as $1.5 \mathrm{eV}[8,21]$.

We have first considered recently proposed, dynamically and mechanically stable single layer group IIVI monolayers due to their peculiar piezoelectric properties. In the case of the FD approach, the 
piezoelectric stress coefficients $\left(e_{11}\right)$ have been determined by calculating the derivative of the change in polarization density with applied uniform strain, $\varepsilon_{11}$ coefficients along the armchair side of the rectangular cell. The results clearly show the expected linear dependence of the polarization to applied strain for both static and relaxed atomic positions as seen in Figure 2. Here, the polarization densities have been obtained by dividing the electronic and ionic dipole moments calculated by VASP with the in-plane area of the unit cell of considered two-dimensional materials, see Figure 1. Therefore, the unit of polarization and also $e_{11}$ coefficient is $\mathrm{Cm}^{-1}$ for these layered materials unlike the unit in threedimensional crystal case, $\mathrm{Cm}^{-2}$.

(a) Clamped Ion

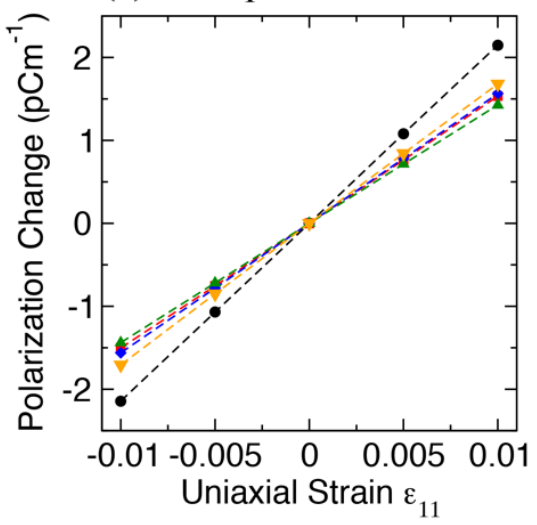

(b) Relaxed Ion

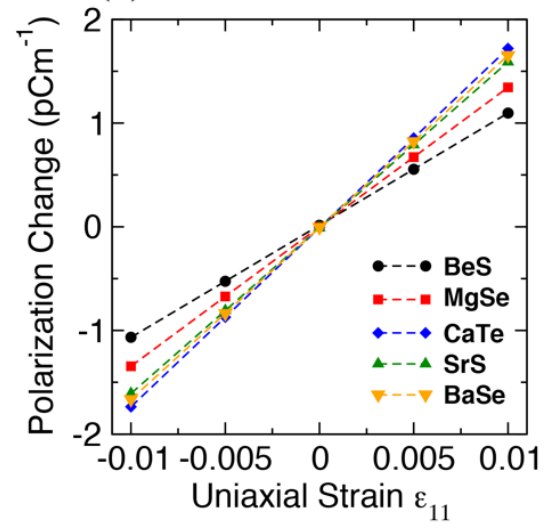

Figure 2. (a) Clamped-ion and (b) relaxed-ion polarization change under applied uniaxial strain $\left(\varepsilon_{11}\right)$ along the $\boldsymbol{x}$ direction for the selected materials. Piezoelectric coefficient is determined from the slope of the curves.

Afterwards, DFPT based calculations which directly gives the electronic and ionic contribution of piezoelectric tensor have been carried out. In order to obtain the coefficients representing the twodimensional case the resulting tensor components in unit of $\mathrm{Cm}^{-2}$ have been divided by the length of the unit cell along the out-of-plane direction $(z)$. Following the FD and DFPT based calculations, $d_{11}$ coefficients, which is a measure of the mechanical-electrical energy conversion ratio and comparable with three dimensional crystals, are determined via Eq. 3 for both methods.

$e_{11}$ and $d_{11}$ coefficients for 15 group II-VI monolayers, predicted by both FD and DFPT approaches are depicted in Figure 3. The difference between the values obtained with the two methods are not significant when only the clamped ion (purely electronic) part considered as seen as blue bars in Figure 3. However, the results differ significantly when the relaxed ion coefficients (both electronic and ionic contribution) considered, see yellow bars in Figure 3. Moreover, the difference between $e_{11}$ coefficients calculated by FD and DFPT are larger for the materials with the smaller coefficients. Recently, Jong et $a l$. [1] have calculated the piezoelectric properties of nearly a thousand compounds by VASP based on DFPT approach and compared their results with available experimental data to establish the accuracy. Their results clearly indicate the accuracy of the DFPT on piezoelectric coefficient calculations and therefore, possibility of error in relaxed-ion $e_{11}$ coefficient calculations with FD approach. In order to test this conclusion, we have performed calculations for $2 \times 2 \times 1$ and $3 \times 3 \times 1$ super-cell structures of selected materials with both methods. We have predicted that the ionic contribution calculated by FD approach changes with the considered crystal size in polarization calculations due to the relaxation of atoms to the positions with different symmetry for different crystal sizes. However, exactly the same results for different conventional cell sizes and symmetries have been obtained with DFPT calculations.

When the results calculated by DFPT are considered as a reference, the electronic and ionic polarization of these materials have opposite sign and the magnitude of the ionic contribution is larger than the electronic contribution for all the considered materials except those with Be atom (relaxed ion 
contribution is equal to the sum of electronic plus ionic contributions). Despite the explicit difference in the coefficients calculated with two different approaches, our results clearly show that the piezoelectric potentials of the considered materials can be determined by both methods. For instance, the $d_{11}$ values predicted for $\mathrm{Mg}, \mathrm{Ca}, \mathrm{Sr}$, and $\mathrm{Ba}$ based materials clearly indicate a great potential of these materials for future piezoelectric applications. Even the values calculated with DFPT (between 7 and $27 \mathrm{pmV}^{-1}$ ), that are smaller than the ones predicted with FD, are quite larger than the bulk materials including $\alpha$-quartz [22], wurtzite-GaN, and wurtzite-AlN [23] which are widely used in industry. Although the relaxed-ion $e_{11}$ coefficients of these materials span a limited range $\left(0.07-1.24 \mathrm{pCm}^{-1}\right)$, the absolute values of the relaxed-ion $d_{11}$ coefficients vary over a much larger range as $0.15-27 \mathrm{pm} / \mathrm{V}$.

(a) FD

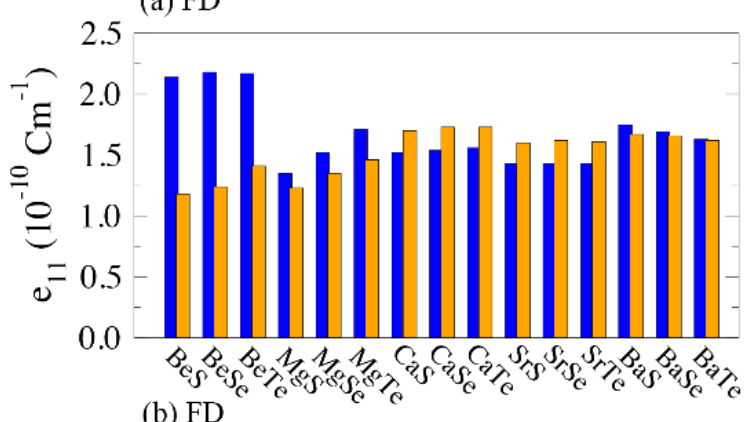

(b) FD

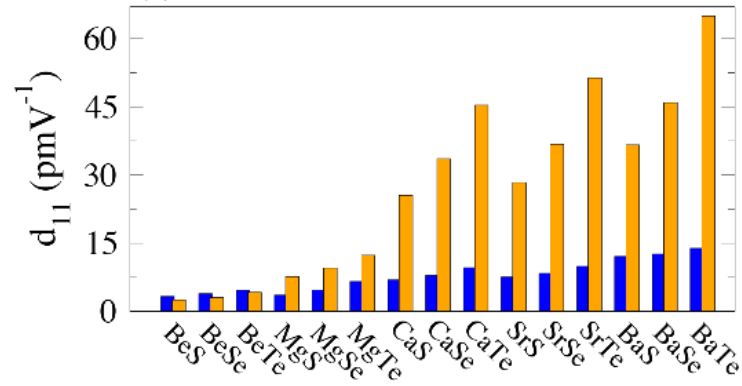

(c) DFPT

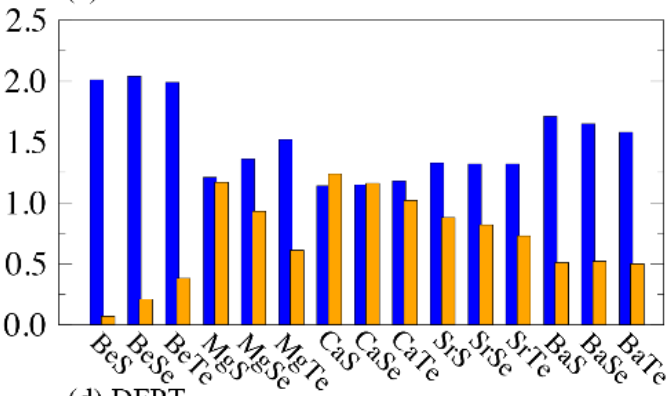

(d) DFPT

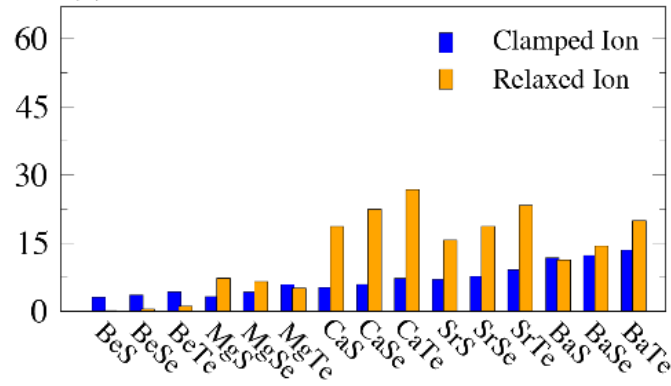

Figure 3. Clamped- and relaxed-ion piezoelectric stress $\left(e_{11}\right)$ and piezoelectric strain $\left(d_{11}\right)$ coefficients of hexagonal group II-VI monolayer materials calculated by (a - b) FD and (c - d) DFTB approaches. Here, blue and Yellow bars depict the clamped-ion and relaxed-ion piezoelectric coefficients, respectively.

The difference in calculated relaxed-ion piezoelectric coefficients, predicted for group II-VI planar single layer crystals, are materials specific and have no distinct dependency on any physical quantity such as lattice constants, electronic structure, pseudopotential, and amount of applied strain. Therefore, we compare these two methods for another well-known two dimensional structures, transition metal dichalcogenides that have non-planar crystal structures. As seen in Figure 4, our results for selected TMDCs resemble nearly the same futures with the ones predicted for group II-VI planar crystals. Similarly, the notable difference between the values obtained by two methods arising from the ionic contribution calculation of FD approach have been observed. However, the variance between the relaxed-ion coefficients predicted with these methods are not as much as that of obtained for group II-VI monolayers. The periodic trends determined by both approaches (the relaxed ion coefficients decrease when moving downward in the group of transition metal elements (i.e., from $\mathrm{Cr}$ to $\mathrm{W}$ ) and increase when moving downward in the group of chalcogenide elements) are exactly the same. Apart from the difference between the results generated by the two methods, the values are in good agreement with that previously published [7] and the predicted relaxed-ion $e_{11}$ coefficients of single layer $\mathrm{MoS}_{2}$ (FD: $4.91 \times 10^{-10} \mathrm{Cm}^{-1}$ and DFTB: $3.62 \times 10^{-10} \mathrm{Cm}^{-1}$ ) are comparable with the experimentally measured value, $2.90 \times 10^{-10} \mathrm{Cm}^{-1}[12]$.

When the results calculated by DFPT are considered as a reference, the electronic and ionic polarization of these materials have opposite sign and the magnitude of the ionic contribution is larger than the electronic contribution for all the considered materials except those with Be atom (relaxed ion 
contribution is equal to the sum of electronic plus ionic contributions). Despite the explicit difference in the coefficients calculated with two different approaches, our results clearly show that the piezoelectric potentials of the considered materials can be determined by both methods. For instance, the $d_{11}$ values predicted for $\mathrm{Mg}, \mathrm{Ca}, \mathrm{Sr}$, and $\mathrm{Ba}$ based materials clearly indicate a great potential of these materials for future piezoelectric applications. Even the values calculated with DFPT (between 7 and $27 \mathrm{pmV}^{-1}$ ), that are smaller than the ones predicted with FD, are quite larger than the bulk materials including $\alpha$-quartz [22], wurtzite-GaN, and wurtzite-AlN [23] which are widely used in industry. Although the relaxed-ion $e_{11}$ coefficients of these materials span a limited range $\left(0.07-1.24 \mathrm{pCm}^{-1}\right)$, the absolute values of the relaxed-ion $d_{11}$ coefficients vary over a much larger range as $0.15-27 \mathrm{pm} / \mathrm{V}$.
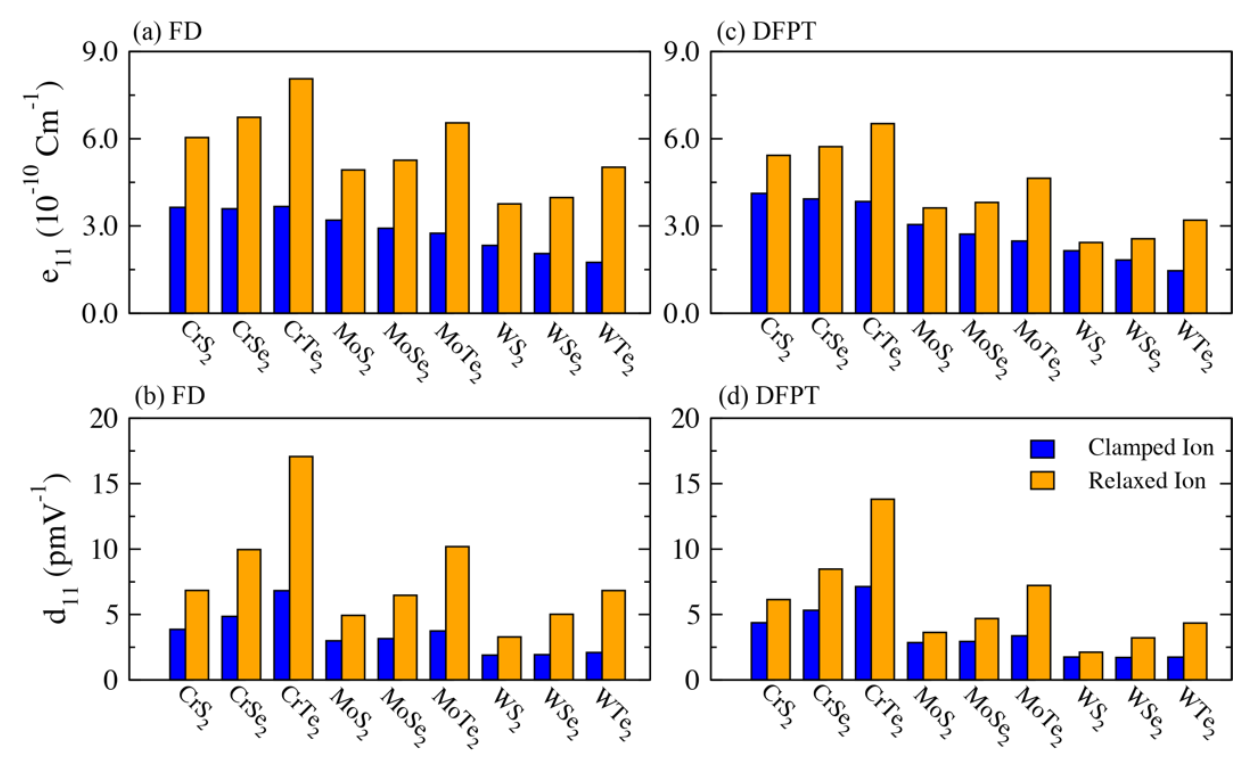

Figure 4. Clamped- and relaxed-ion piezoelectric stress $\left(e_{11}\right)$ and piezoelectric strain $\left(d_{11}\right)$ coefficients of hexagonal TMDC monolayer materials calculated by (a - b) FD and (c - d) DFTB approaches. Here, blue and Yellow bars depict the clamped-ion and relaxed-ion piezoelectric coefficients, respectively.

\section{CONCLUSIONS}

In summary, well known finite difference and density functional perturbation theory based piezoelectric property calculation methods have been compared considering two different single layer two-dimensional material families, group II-VI and transition metal dichalcogenides. After series of test calculations (in particular considering different cell sizes in polarization calculations), the error in finite difference based ionic contribution calculations have been observed for some materials. However, our results clearly show that the piezoelectric potential of two dimensional materials can be determined by using both methods.

\section{ACKNOWLEDGEMENTS}

This study was supported by Anadolu University Scientific Research Projects Commission under the grant no:1605F368. 


\section{REFERENCES}

[1] de Jong M, Chen W, Geerlings H, Asta M, Persson KA. A database to enable discovery and design of piezoelectric materials. Scientific Data 2015; 2: 150053

[2] Morten, B, De Cicco G, Prudenziati M. Resonant pressure sensor based on piezoelectric properties of ferroelectric. Thick Films. Sens. Actuators A 1992; 31: 153-158.

[3] Jaffe H. Berlincourt DA, Piezoelectric transducer. Materials. Proc. IEEE 1965; 53: 1372-1386.

[4] Wang ZL, Song JH. Piezoelectric Nanogenerators Based on Zinc Oxide Nanowire Arrays. Science 2006; 312: 242-246.

[5] Lopez-Suarez M, Pruneda M, Abadal G, Rurali R. Piezoelectric Monolayers as Nonlinear Energy Harvesters. Nanotechnology 2014; 25: 175401-175405.

[6] Wu W, Wang L, Li Y, Zhang F, Lin L, Niu S, Chenet D, Zhang X, Hao Y, Heinz TF, Hone J, Wang ZL. piezoelectricity of single-atomic-layer $\mathrm{MoS}_{2}$ for energy conversion and piezotronics. Nature 2014; 514: 470-474.

[7] Blonsky MN, Zhuang HL, Singh AK, Hennig RG. Ab initio prediction of piezoelectricity in twodimensional materials. ACS Nano 2015; 9: 9885-9891.

[8] Alyörük MM, Aierken Y, Çakır D, Peeters FM, Sevik C. Promising piezoelectric performance of single layer transition-metal dichalcogenides and dioxides. J Phys Chem C, 2015; 119: 2323123237.

[9] Fei R, Li W, Li J, Yang L. Giant Piezoelectricity of Monolayer Group IV Monochalcogenides: SnSe, SnS, GeSe, and GeS. Appl Phys Lett 2015; 107: 173104-173108.

[10] Li W, Li J. Piezoelectricity in Two-Dimensional Group-III Monochalcogenides. Nano Res. 2015; 8: 3796-3802.

[11] Gomes LC, Carvalho A, Castro Neto A. H. Enhanced Piezoelectricity and Modified Dielectric Screening of Two-Dimensional Group-IV Monochalcogenides. Phys Rev B 2015; 92: 214103.

[12] Zhu H, Wang Y, Xiao J, Liu M, Xiong S, Wong ZJ, Ye Z, Ye Y, Yin X, Zhang X. Observation of piezoelectricity in free- standing monolayer $\mathrm{MoS}_{2}$. Nat Nanotechnol, 2014; 10: 151-155.

[13] Sevik C, Çakır D, Gülseren O, Peeters FM. Peculiar piezoelectric properties of soft twodimensional materials. J Phys Chem C 2016; 120: 13948-13953.

[14] Duerloo KAN, Ong MT, Reed EJ, Intrinsic Piezoelectricity in Two-Dimensional Materials. J Phys Chem Lett. 2012; 3: 2871-2876.

[15] Nunes RW, Gonze X. Berry-Phase Treatment of the Homogeneous Electric Field Perturbation in Insulators. Phys Rev B 2001; 63: 155107.

[16] Kresse G, Hafner J. Ab Initio Molecular Dynamics for Liquid Metals. Phys. Rev. B. 1993; 47: 558-561.

[17] Wu X, Vanderbilt D, Hamann DR. Systematic treatment of displacements, strains and electric fields in density-functional perturbation theory. Phys Rev B. 2005; 72: 035105. 
Sevik / Anadolu Univ. J. of Sci. and Technology A-Appl. Sci. and Eng. 18 (3) - 2017

[18] Perdew JP, Burke K, Ernzerhof M. Generalized Gradient Approximation Made Simple. Phys Rev Lett 1996; 77: 3865-3868.

[19] Monkhorst HJ, Pack JD. Special Points for Brillouin-Zone Integrations. Phys Rev B 1976; 13: 5188-5192.

[20] Wu X, Vanderbilt D, Hamann, DR. systematic treatment of displacements, strains and electric fields in Density-functional perturbation theory. Phys Rev B 2005; 72: 035105.

[21] Zheng H, Li XB, Chen NK, Xie SY, Tian WQ, Chen Y, Xia H, Zhang SB, Sun HB. Monolayer IIVI Semiconductors: A First-Principles Prediction. Phys Rev B 2015; 92: 115307.

[22] Behmann R, Elastic and Piezoelectric Constants of alpha-Quartz. Phys. Rev. 1958; 110: 1060-1061.

[23] Lueng CM, Chan HL W, Surya C, Choy CL. Piezoelectric coefficient of aluminum nitride and Gallium Nitride. J Appl Phys 2000; 88: 5360-5363. 